\title{
Adaptive Pole Assignment by State Feedback.
}

\author{
J.W. Polderman \\ Centre for Mathematics and Computer Science \\ P.O. Box 4079, 1009 AB Amsterdam. The Netherlands
}

\begin{abstract}
An algorithm for adaptive pole placement for a restricted class of systems is proposed. The asymptotic properties of the algorithm are analysed by studying the invariant points and the asymptotic active part of the state space. A weak form of self-tuning is derived

1980 Mathematics Subject Classification: $93 \mathrm{C} 40$

Key Words \& Phrases: Adaptive pole-placement, self-tuning, certainty-equivalence.
\end{abstract}

\section{l. INTRODUCTION.}

This note is concerned with the problem of adaptive pole placement of deterministic systems without external excitation. We consider a linear plant with only one input and observed state. The problem we then want to study is the asymptotic assignment of the closed-loop poles in a pre-described configuration by means of adaptive state feedback. The proposed algorithm is based on direct estimation of the plant-parameters and the certainty- equivalence principle. Since identification takes place in closed-loop the true system cannot be identified without external excitation. However it will be shown that because of the chosen control objective, closed-loop identification causes no extra difficulties, which is in contrast with adaptive LQ control (see [4]).

Since no external excitation is added, it cannot be expected that the state trajectory will span the whole state-space. Therefore the concept of excitation subspace will be introduced to analyse the proposed algorithm.

This work is motivated by two approaches of adaptive stabilization that appeared in the literature. The first is the model reference adaptive control method (see for instance [5]). The other approach has been presented in a series of papers which culminated in [2]. The first method was developed for systems in input/output form, whereas the second works in state space. In both cases stability results are derived without imposing conditions on exciting signals. Here we make an attempt to derive a weak form of self-tuning.

A shorter version of this paper is [3]

A serious difficulty is caused by the fact that we consider systems in state space form and try to identify the $(A, b)$ parameters. During the estimation procedure all estimates have to be reachable in order to be able to calculate the control law to be applied. This problem has not yet been solved and will be commented upon elsewhere in the paper.

We start with a description of the class of systems under consideration and of the control problem. Next we present our algorithm. We will then formulate our main theorem followed by its proof, which is distributed over several lemmata. We end with some concluding remarks. 
2. Preliminaries.

Consider the following system:

$$
x_{k+1}=A x_{k}+b u_{k},
$$

where $(A, b) \in E:=\left\{(A, b) \in \mathbb{R}^{n \times n} \times \mathbb{R}^{n \times 1} \mid(A, b)\right.$ reachable $\}$.

Let $\Lambda:=\left\{\lambda_{1}, \ldots, \lambda_{n}\right\} \subset \mathbb{C}$ be such that $\lambda \in \Lambda \Rightarrow \bar{\lambda} \in \Lambda$. Define $\sigma \in \mathbb{R}[X]$ by: $\sigma(X)=\prod_{1}^{n}\left(X-\lambda_{t}\right)$.

Define $f: E \rightarrow \mathbb{R}^{1 \times n}$ by:

$$
f(A, b):=-[0 \ldots 01]\left[b \vdots b A \vdots \ldots \ldots A^{n-1}\right]^{-1} \sigma(A)
$$

Then the characteristic polynomial of $A+b f(A, b)$ is exactly $\sigma$ and moreover since the system is single-input $f(A, b)$ is the only feedback law with that property. (see [6]).

Suppose now that the true value, say $\left(A_{0}, b_{0}\right)$ of the system-parameters is not known, then the question arises how 'much' we should know about them to be able to control the system as desired. Of course it will be enough to know $f\left(A_{0}, b_{0}\right)$, but we will see that this is not the minimum of information we need.

In this paper we want to present an algorithm based on direct estimation of $\left(A_{0}, b_{0}\right)$ and the certainty equivalence principle. This structure causes certain identification problems (see [4]). In the following theorem the best possible situation for an estimate $(A, b)$ is studied.

Theorim 2.1 Let $(A, b) \in E$ and $\mathbb{V}$ a linear subspace of $\mathbb{R}^{n \times n}$ such that:

i) For all $v \in \mathbb{V}:\left(A_{0}+b_{0} f(A, b)\right) v \in \mathfrak{V}$

ii) For all $v \in \mathcal{V}:\left(A_{0}+b_{0} f(A, b)\right) v=(A+b f(A, b)) v$

Then:

For all $v \in \vee V: f(A, b) \nu=f\left(A_{0}, b_{0}\right) v$.

PROOF: Suppose that $\Lambda \subset \mathbb{R}$ and that $\lambda_{l} \neq \lambda_{j}$ for all $i \neq j$. Let $\checkmark$ be one-dimensional. Then $\checkmark$ is generated by an eigenvector $v$ of $(A+b f(A, b))$ corresponding to let's say $\lambda:=\lambda_{1}$. Hence $\left(A_{0}+b_{0} f(A, b)\right) v=\lambda v$. Suppose $\left(A_{0}, b_{0}\right)$ is in standard controllable form. Then $v=\left[1, \lambda, \ldots, \lambda^{n} " 1\right]^{T}$. Since $\lambda$ is an eigenvalue of $\left(A_{0}+b_{0} f\left(A_{0}, b_{0}\right)\right)$, there exists $\tilde{v}$ such that $\left(A_{0}+b_{0} f\left(A_{0}, b_{0}\right)\right) \tilde{v}=\lambda \tilde{v}$. It is easy to see that $\nu=\mu \tilde{\nu}$, for some $\mu \neq 0$. Hence $\left(A_{0}+b_{0} f\left(A_{0}, b_{0}\right)\right) v=\left(A_{0}+b_{0} f(A, b)\right) \nu$. Since $b_{0} \neq 0$, we conclude that $f(A, b) v=f\left(A_{0}, b_{0}\right) v$.

If $\operatorname{dim}^{\prime} \gamma>1$, then " $\nabla$ has a basis of eigenvectors and the above reasoning gives the result. For general $\Lambda$ the proof goes along the same lines, but then one has to study several different cases. We skip the details.

Commint. Suppose we have an estimate $(A, b)$ of $\left(A_{0}, b_{0}\right)$, according to the certainty equivalence principle we will then apply $u_{k}=f(A, b) x_{k}$. The resulting closed -loop system is:

$$
\left.x_{k+1}=A_{0}+b_{0} f(A, b)\right) x_{k}
$$

Whereas on the basis of our guess we would predict:

$$
\hat{x}_{k+1}=(A+b f(A, b)) x_{k}
$$

Suppose now that for all $k$ we have $\hat{x}_{k+1}=x_{k+1}$, this is in some sense the best situation we could have. For once we have an estimate $(A, b)$ with that property, the observed data will not give rise to any update of the parameter estimates. Define $V:=\operatorname{span}\left\{x_{k}\right\}$, then it can be checked that $V$ satisfies the conditions of Theorem 2.1 and hence we conclude that for all $v \in V, f(A, b) v=f\left(A_{0}, b_{0}\right) v$. In particular: $f(A, b) x_{k}=f\left(A_{0}, b_{0}\right) x_{k}$, for all $k$, or otherwise stated the applied input equals the desired 
input.

Summarizing: In order to control the system (2.1) as desired, it is not necessary to know $\left(A_{0}, b_{0}\right)$, nor is it necessary to know $f\left(A_{0}, b_{0}\right)$, we only need to know the action of $f\left(A_{0}, b_{0}\right)$ on the active part of the state space.

3. DisCRIPTION OF THE Algorithim.

We will introduce the algorithm inductively. Choose the initial guess $\left(\hat{A}_{0}, \hat{b}_{0}\right) \in E$ of $\left(A_{0}, b_{0}\right)$ arbitrarily. Suppose the $k$-th guess $\left(\hat{A}_{k}, \hat{b}_{k}\right)$ has been calculated. Then take $u_{k}=f\left(\hat{A}_{k}, \hat{b}_{k}\right) x_{k}$. This gives :

$$
x_{k+1}=\left(A_{0}+b_{0} f\left(\hat{A}_{k}, \hat{b}_{k}\right)\right) x_{k}
$$

Define

$$
\hat{G}_{k+1}:=\left\{(A, b) \mid\left(A+b f\left(\hat{A}_{k}, \hat{b}_{k}\right)\right) x_{k}=x_{k+1}\right\}
$$

$\hat{G}_{h+1}$ is an affine subvariety of $\mathbb{R}^{n \times n+n \times 1}$. Hence we can take $\left(\hat{A}_{k+1}, \hat{b}_{k+1}\right)$ to be the orthogonal projection of $\left(\hat{A}_{k}, \hat{b}_{k}\right)$ on $\hat{G}_{k+1}$ in $\mathbb{R}^{n \times n+n \times 1}$. This procedure is equivalent to the following recursion for $\left(A_{h}, \hat{b}_{k}\right)$ :

$$
\begin{aligned}
\hat{A}_{k+1} & =\hat{A}_{k}+\left(\left\|u_{k}\right\|^{2}+\left\|x_{k}\right\|^{2}\right)^{-1}\left(x_{k+1}-\hat{x}_{k+1}\right) x_{k}^{T} \\
\hat{b}_{k+1} & =\hat{b}_{k}+\left(\left\|u_{k}\right\|^{2}+\left\|x_{k}\right\|^{2}\right)^{-1}\left(x_{k+1}-\hat{x}_{k+1}\right) u_{k} \\
u_{k} & =f\left(\hat{A}_{k}, \hat{b}_{k}\right) x_{k} \\
\hat{x}_{k+1} & =\left(\hat{A}_{k}+\hat{b}_{k} f\left(\hat{A}_{k}, \hat{b}_{k}\right)\right) x_{k}
\end{aligned}
$$

COMment. The algorithm is based on two ideas. The first is concerned with the analysis of the invariant points of the algorithm. From the above description it follows that $\left(\hat{A}_{k+1}, \hat{b}_{k+1}\right)=\left(\hat{A}_{k}, \hat{b}_{k}\right)$ if and only if $\left(\hat{A}_{k}, \hat{b}_{k}\right) \in \hat{G}_{k}+1$. Define $G:=\left\{(A, b) \mid A+b f(A, b)=A_{0}+b_{0} f(A, b)\right\}$. Then certainly every element of $G$ is an invariant point of 3.2. It follows from Theorem 2.1 that $(A, b) \in G$ implies $f(A, b)=f\left(A_{0}, b_{0}\right)$. Hence if all the limit points of $\left\{\left(\hat{A}_{k}, \hat{b}_{k}\right)\right\}_{k \in N}$ are in $G$, then we have achieved our control objective.

The second motivation is the following. Suppose at time $k$ we have the estimate $\left(\hat{A}_{k}, \hat{b}_{k}\right)$ of $\left(A_{0}, b_{0}\right)$. The certainty equivalence principle tells us to act as if we were sure about $\left(A_{0}, b_{0}\right)$ and hence we should apply $u_{k}=f\left(\hat{A}_{k}, \hat{b}_{k}\right) x_{k}$ to the real system. After having done so we observe the new state $x_{k}+1$. Now $\hat{G}_{k+1}$ is exactly the set of those parameters $(A, b)$ that are able to explain the observed data $\left(x_{k}, x_{k+1}, u_{k}\right)$. Since obviously $\left(A_{0}, b_{0}\right) \in \hat{G}_{k+1}$, it is natural to choose $\left(\hat{A}_{k+1}, \hat{b}_{k+1}\right)$ somewhere in $\hat{i}_{k}+1$. The reason that we take the orthogonal projection of $\left(\hat{A}_{k}, \hat{b}_{k}\right)$ on $\hat{G}_{k+1}$ is that as a direct consequence $\left\|\left(A_{0}, b_{0}\right)-\left(\hat{A}_{k}, \hat{b}_{k}\right)\right\|$ converges. The idea of orthogonality was already used in [1], where it was derived from a certain stochastic approximation algorithm. Here we choose it as a starting point rather than as a consequence.

One further remark has to be made. The algorithm 3.2 only makes sense if $\left(\hat{A}_{k}, \hat{b}_{k}\right)$ is reachable for every $k \in \mathbb{N}$. Throughout the paper we will hence make the following assumptions: For all $k \in \mathbb{N}$, $\left(\hat{A}_{k}, \hat{b}_{k}\right) \in E$, and also all limit points of $\left\{\left(\hat{A}_{k}, \hat{b}_{k}\right)\right\}_{k \in N}$ are in $E$. The first assumption is not really a limitation, for it is not difficult to see that for a generic choice of $\left(\hat{A}_{0}, \hat{b}_{0}\right) \in E,\left(\hat{A}_{k}, \hat{b}_{k}\right) \in E$ for all $\mathrm{k}$. The condition on the limit points however is undesirable and should follow as a consequence of the first. This point is still under investigation.

4. ANAI.YSIS OF THE ALGORITHM.

The properties of the algorithm will be derived in several steps. We will need some definitions and lemmata before we can draw asymptotic conclusions. First we shall state our main result. 
THRorim 4.1 Consider the (controlled) system $(2.1,3.2)$, there exists a sequence of matrices $\left\{\Delta_{k}\right\}_{k, N}$. such that:

$$
\text { i) } \begin{aligned}
x_{k+1} & =\left(A_{0}+b_{0} f\left(\hat{A}_{k}, \hat{b}_{k}\right)\right) x_{k} \\
& \left.=\left(A_{0}+b_{0} f\left(A_{0}, b_{0}\right)+\Delta_{k}\right) x_{k}\right)
\end{aligned}
$$

ii) $\lim _{k \rightarrow \infty} \Delta_{k}=0$

COMment. Theorem 4.1 tells us that asymptotically the action of the closed-loop matrix is identical (1) that of the optimal closed-loop matrix. It should be noticed that we do not claim that the real closed-loop matrix converges to the optimal one, but only as far as the action on the real statetrajectory is concerned. This weaker form of convergence is not surprising if we realise the fact that the estimation procedure only receives information about the action of the real closed-loop matrix on the state-trajectory. We propose the term 'weak self-tuning' for this kind of behaviour. Self-tuning would have implied that $\lim _{h \rightarrow \infty}\left(A_{0}+b_{0} f\left(\hat{A}_{k}, \hat{b}_{h}\right)-A_{0}+B_{0} f\left(A_{0}, b_{0}\right)\right.$, which we do not claim.

We shall now state two technical lemmata which we will need in the proof of Theorem 4.1.

I. ImMA 4.2 Let $K \subset \mathbb{R}^{n \times n}$ be compact and let $\epsilon>0$. Then there exists $\gamma>0$ such that for all $A \in K$ and for all $x \in \mathbb{R}^{n}$ with $\|A x\| \geqslant \epsilon$ and $x^{T} x=1:\left\|A . x x^{T}\right\| \geqslant \gamma$.

Proof Suppose the claim is not true. Then there exist $A \in K$ and $x \in \mathbb{R}^{n}$ with $\|A x\| \geqslant \epsilon, x^{T} x=1$ and $\left\|A x x^{T}\right\|=0$. This implies that $A x x^{T}=0$, which means that either $A x$ or $x^{T}=0$, which are both contradictions.

Li:mMA 4.3 Let $\left\{M_{k}\right\}_{k} \cdot \mathbf{N}$ be a bounded sequence of matrices in $\mathbb{R}^{n \times n}$, such that $\lim _{k \rightarrow \infty}\left\|M_{k+1}-M_{k}\right\|=0$. Let $x_{0} \in \mathbb{R}^{n}$ be given and define the sequence $\left\{x_{k}\right\}$ by putting: $x_{k+1}=M_{k} x_{k}$. Suppose $\lim _{h \rightarrow \infty} M_{t_{4}}=M$, define : $x$ as the linear subspace generated by the limit points of $x_{i+t_{4}}$, where $l$ ranges from 0 to infinity. Then $M: x<$.

PRoOF Suppose $x^{*}$ is a limit point of $\left\{x_{i+t_{k}}^{*}\right\}$ for some l. Say $\lim _{k \rightarrow \infty} x_{i+s_{k}}^{*}=x^{*}$, for some subsequence $\left\{s_{k}\right\}$ of $\left\{t_{k}\right\}$. Then:

$$
\begin{aligned}
& M x^{*}=\lim _{k \rightarrow \infty} M_{l+s_{k}} x_{l+l_{k}}=\lim _{k \rightarrow \infty} \frac{1}{\left\|x_{l}+s_{k}\right\|} M_{l+s_{\star}} x_{l+s_{A}}=\lim _{k \rightarrow \infty} \frac{1}{\left\|x_{l}+s_{4}\right\|} x_{l+1+s_{k}} \\
& =\lim _{k \rightarrow \infty} \frac{\left\|x_{l}+1+s_{k}\right\|}{\left\|x_{l}+s_{A}\right\|} x_{i+l+s_{A}}=\lim _{k \rightarrow \infty} \frac{\left\|M_{l}+s_{k} x_{l+s_{k}}\right\|}{\left\|x_{l}+s_{\mathrm{A}}\right\|} x_{i+l+s_{k}}=\lim _{k \rightarrow \infty}\left\|M_{l+s_{k}} x_{i+s_{\mathrm{A}}}\right\| x_{i+1+s_{A}} \\
& =\left\|M x^{*}\right\| \lim _{k \rightarrow \infty} x_{i+1+s_{i}} .
\end{aligned}
$$

Hence $M x^{*} \in X$. By linearity the result follows.

LEmma $4.4\left\|\left(\hat{A}_{h}, \hat{b}_{k}\right)-\left(A_{0}, b_{0}\right)\right\|$ is a decreasing sequence, hence it converges to some real constant $R \geqslant 0$.

PR()OF This a direct consequence of the orthogonal projection feature which assures that $\left\|\left(\hat{A}_{h}, \hat{b}_{k}\right)-\left(A_{0}, b_{0}\right)\right\| \geqslant\left\|\left(\hat{A}_{k+1}, \hat{b}_{k+1}\right)-\left(A_{0}, b_{0}\right)\right\|$. 
Although Lemma 4.4 is very simple not to say trivial, it is the central feature of our algorithm. A direct consequence of 4.4 is that $\left(\hat{A}_{k}, \hat{b}_{k}\right)$ converges to a sphere with centre $\left(A_{0}, b_{0}\right)$ and radius $R$. If $R=0$ then $\left(A_{h}, \hat{b}_{k}\right) \rightarrow\left(A_{0}, b_{0}\right)$ and we are done. In the sequel we shall hence assume that $R>0$.

DiFINITION 4.5

i) Denote by $\left\{\left(\bar{A}_{1}, \bar{b}_{1}\right)\right\}_{1, I}$ the set of limit points of $\left\{\left(\hat{A}_{k}, \hat{b}_{k}\right)\right\}_{k, \mathbf{N}}$. Assume that for every $i \in I$ $\lim _{h \rightarrow \infty}\left(\hat{A}_{i_{i}}, \hat{b}_{i^{\prime}}\right)=\left(\hat{A}_{i}, \hat{b}_{t}\right)$. Since $\left(\hat{A}_{k}, \hat{b}_{k}\right)$ cannot make positive jumps bounded from below infinitely $h \rightarrow \infty$
often without penetrating the sphere to which it was supposed to converge from the outside, $I$ is either a singleton or an infinite set.

ii) Let $\left\{x_{k}\right\}_{k}, \mathbf{N} \subset \mathbb{R}^{n}$ be the state trajectory of the real closed-loop system. Define for every $x \in \mathbb{R}^{n}$ : $x^{*}:=x /\|x\|$ if $x \neq 0$, and $0^{*}:=0$. Denote by $x$ the linear subspace of $\mathbb{R}^{n}$ generated by the limit points of $\left\{x_{k}^{*}\right\}$.

iii) Let for every $i \in I, X_{i}$ be the subspace generated by the limit points of $x_{i+t_{k}^{*}}^{*}$, where $l$ ranges from zero to infinity.

The space $x$ can be viewed as the excitation subspace of the state space. It reveals the separation between fast and slow convergence/divergence. Since it is easy to see that 3.2 depends only on $x_{k}$ rather then on $x_{k}$ itself, it will appear that $\chi$ will be very helpful in the analysis of the algorithm. It will be supposed that $\operatorname{dim} x>0$, since if $\operatorname{dim} x=0$ then $x_{k}^{*}=0$ for $k \geqslant k_{0}$ for some $k_{0}$ and then there is very little to analyse.

$\forall$ can be interpreted as the excitation space belonging to $\left(\bar{A}_{i}, \bar{b}_{i}\right)$. The reason that we take the union over all $l$, is that since $\left\|\left(\hat{A}_{k+1}, \hat{b}_{k+1}\right)-\left(\hat{A}_{k}, \hat{b}_{k}\right)\right\| \rightarrow 0, \lim _{k \rightarrow \infty}\left(\hat{A}_{l+i_{k}^{\prime}}, \hat{b}_{l+i_{k}}\right)=\left(\bar{A}_{i}, \bar{b}_{i}\right)$, for all $l$.

LiMMA $4.6 \sum_{i=1} x_{i}=x$

Proof It is obvious that the left hand side is contained in the right hand side.

Suppose $x^{*}$ is a limit point of $\left\{x_{k} \dot{k}\right\}$, say, $\lim _{k \rightarrow \infty} x_{s_{k}}^{*}=x^{*}$. Let for some $i \in I,\left(\bar{A}_{i}, \bar{b}_{i}\right)$ be a limit point of $\left(\hat{A}_{1}, \hat{b}_{s_{1}}\right)$, then $x^{*} \in X_{2}$. For arbitrary $x \in \mathcal{X}$ the result follows by writing $x$ as a linear combination of limit points.

L.I:MmA $4.7 \lim _{k \rightarrow \infty}\left[\left(\hat{A}_{k}+\hat{b}_{k} f\left(\hat{A}_{k}, \hat{b}_{k}\right)\right)-\left(A_{0}+b_{0} f\left(\hat{A}_{k}, \hat{b}_{k}\right)\right)\right] \dot{x}_{k}^{*}=0$.

Proof Define $M_{k}:=\left[\left(\hat{A}_{k}+\hat{b}_{k} f\left(\hat{A}_{k}, \hat{b}_{\dot{k}}\right)\right)-\left(A_{0}+b_{0} f\left(\hat{A}_{k}, \hat{b}_{k}\right)\right)\right]$. Suppose the claim is not true. Then there exists $\epsilon>0$ and a sequence $\left\{s_{k}\right\}$ such that: $\left\|M_{s_{k}}\right\| \geqslant \epsilon$ for all $k$. From $3.2 \mathrm{a}$ we see that:

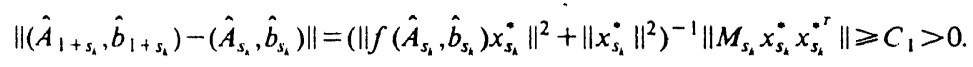

This follows from the facts that $\left(\hat{A}_{k}, \hat{b}_{k}\right)$ is bounded and reachable, the continuity of $f$ on $E$, the reachability of $\left(\bar{A}_{i}, \bar{b}_{i}\right)$ and Lemma 4.2. Now denote $\left\|\left(\hat{A}_{k}, \hat{b}_{k}\right)-\left(A_{0}, b_{0}\right)\right\|$ by $r_{k}$. Choose $\delta>0$ and let $k_{0}$ be such that $R \leqslant r_{s_{k}} \leqslant R+\delta$ for all $k \geqslant k_{0}$. Using Pythagoras' theorem we see that for all $k \geqslant k_{0}$ :

$$
r_{s_{k}}-r_{1+s_{4}} \geqslant r_{s_{4}}-\left(r_{s_{4}}^{2}-C_{1}^{2}\right)^{1 / 2} \geqslant R\left(1-\left(1-\left(\frac{C_{1}}{R+\delta}\right)^{2}\right)^{1 / 2}\right) \geqslant C_{2}>0 .
$$

Since $r_{k}$ is non-increasing we have $r_{s_{k}}-r_{s_{k+1}} \geqslant C_{2}$, which yields:

$$
r_{s_{4}}<r_{s_{k_{0}}}-C_{2}\left(k-k_{0}\right) \leqslant R+\delta-C_{2}\left(k-k_{0}\right) \text {. }
$$

Hence there exists $k$ such that $r_{s_{k}}<R$, which is a contradiction. 
Lemma 4.8 For every $i \in I$ and $x \in \mathbb{X}_{i}:\left[\left(\bar{A}_{i}+\bar{b}_{l} f\left(\bar{A}_{i}, \bar{b}_{l}\right)\right)-\left(A_{0}+b_{0} f\left(\bar{A}_{i}, \bar{b}_{l}\right)\right)\right] x=0$.

Proof Suppose $x^{*}$ is a limit point of $x_{i+i_{i}^{*}}$, say $\lim _{h \rightarrow \infty} x_{i+s_{4}^{*}}=x^{*}$, then from Lemma 4.7 we deduce: $\left[\left(\bar{A}_{i}+\bar{b}_{i} f\left(\bar{A}_{i}, \bar{b}_{i}\right)\right)-\left(A_{0}+b_{0} f\left(\bar{A}_{i}, \bar{b}_{i}\right)\right)\right] x^{*}=0$. For arbitrary $x \in \mathcal{X}$ the statement follows by writing $x$ as a (finite) linear combination of limit points.

COROI.IARY 4.9

i) For every $i \in I:\left(A_{0}+b_{0} f\left(\bar{A}_{i}, \bar{b}_{i}\right)\right) \mathbb{X}_{i} \subset \mathfrak{X}_{i}$.

ii) For every $i \in I:\left.f\left(\bar{A}_{i}, \bar{b}_{i}\right)\right|_{: i}=\left.f\left(A_{0}, b_{0}\right)\right|_{\text {ac }}$.

PROOF

i) Take $M_{k}=A_{0}+b_{0} f\left(\hat{A}_{k}, \hat{b}_{k}\right)$ in lemma 4.3. Since $\left\|\left(\hat{A}_{k+1}, \hat{b}_{k+1}\right)-\left(\hat{A}_{k}, \hat{b}_{k}\right)\right\| \rightarrow 0$, we have by the continuity of $f$ on $E$ that $\left\|M_{k+1}-M_{k}\right\| \rightarrow 0$.

ii) From Lemma 4.9 we deduce that $\left.\left(\bar{A}_{i}+\bar{b}_{i} f\left(\bar{A}_{i}, \bar{b}_{i}\right)\right)\right|_{x_{i}}=\left(A_{0}+b_{0} f\left(\bar{A}_{i}, \bar{b}_{i}\right)\right) \mid \varkappa_{i}$. From $\left.i\right)$ we deduce that $\left(A_{0}+b_{0} f\left(\bar{A}_{i}, \bar{b}_{1}\right)\right)_{1} \subset \mathrm{X}_{1} \subset \mathrm{x}_{1}$. The result now follows from Theorem 2.1.

THEOREM 4.10

i) $\lim _{h \rightarrow \infty}\left\|\left(f\left(\hat{A}_{k}, \hat{b}_{k}\right)-f\left(A_{0}, b_{0}\right)\right) x_{k}\right\|=0$.

ii) $\lim _{h \rightarrow \infty}\left[\left(A_{0}+b_{0} f\left(\hat{A}_{k}, \hat{b}_{k}\right)\right)-\left(A_{0}+b_{0} f\left(A_{0}, b_{0}\right)\right)\right] x_{k}^{*}=0$.

PROOF

i) Suppose the claim is not true. Then there exist $\epsilon>0$ and a subsequence $\left\{s_{k}\right\}$ such that $\left\|\left(f\left(\hat{A}_{s_{k}}, \hat{b}_{s_{k}}\right)-f\left(A_{0}, b_{0}\right)\right) x_{s_{k}}^{*}\right\| \geqslant \epsilon$, for all $k$. Choose a subsequence $\left\{\tilde{s}_{k}\right\}$ of $\left\{s_{k}\right\}$ such that $\lim _{k \rightarrow \infty}\left(A_{\bar{s}_{1}}, b_{\bar{s}_{4}}\right)=\left(\bar{A}_{l}, \bar{b}_{l}\right)$ for some $i \in I$ and $\lim _{k \rightarrow \infty} x_{\bar{s}_{k}}=x^{*} \in \mathcal{X}_{2}$. Then by Corollary 4.9ii: $\lim _{k \rightarrow \infty}\left\|\left(f\left(\hat{A}_{\bar{s}_{t}}, \hat{b}_{\bar{s}_{4}}\right)-f\left(A_{0}, b_{0}\right)\right) x_{\dot{s}_{t}}^{\cdot}\right\|=\left\|\left(f\left(\bar{A}_{i}, \bar{b}_{1}\right)-f\left(A_{0}, b_{0}\right)\right) x^{*}\right\|=0$, which is a contradiction. The result follows.

ii) This is now trivial.

We will now prove Theorem 4.1:

Proof of Theorem 4.1: Choose $\epsilon>0$. Denote by $S^{n-1}$ the boundary of the unit sphere in $\mathbb{R}^{n}$, and define $B(x, \delta):=\left\{y \in \mathbb{R}^{n}|| x-y \mid<\delta\right\}$. Let for every $x^{*} \in S^{n-1}, g_{x} \in \mathbb{R}^{1 \times n}$ be such that : $\left|g_{x} \cdot x^{*}\right|=>2 \epsilon$. Define:

$$
O_{x}:=S^{n-1} \cap B\left(x^{*}, \delta\right)
$$

Where $\delta>0$ (depending on $x^{*}$ ) is such that:

$$
x \in O_{x} \cdot \Rightarrow\left|g_{x} \cdot x\right|>\epsilon
$$

Then $\left\{O_{x}\right\}_{x} \cdot S^{n-1}$ forms an open covering of $S^{n-1}$. Since $S^{n-1}$ is compact we conclude that there exist $x_{(1)}^{*}, \ldots, x_{(p)} \in S^{n-1}$, such that $\left\{O_{x_{i, 1}}\right\}_{i=1, \ldots, p}$ covers $S^{n-1}$. Define $K_{i}$ as the closure of $O_{x_{i, 1}}$.

Choose subsequences $\left\{s_{k}^{i}\right\}$ of $\mathbb{N}$ with the following properties:
a) $\bigcup_{i=1}^{p} \bigcup_{k=0}^{\infty}\left\{s_{k}^{i}\right\}=\mathbf{N}$
b) $i \neq j \Rightarrow\left\{s_{k}^{i}\right\}_{k \in N} \cap\left\{s_{k}^{i}\right\}_{k \in N}=\varnothing$
\left. c) (limit points of ${\dot{x_{s}}}_{s_{i}}^{\cdot}\right) \subset K_{i}$

Define $g_{(i)}:=g_{s_{i}^{*}}$. 
Choose $\left(A_{1}, h_{1}\right) \in E$ such that:

$$
A_{0}+b_{0} f\left(A_{0}, b_{0}\right)=A_{1}+b_{1} g_{(1)}
$$

Define:

$$
g_{h}^{\prime}:=b_{i}^{*}\left(A_{0}-A_{1}+b_{0} f\left(\hat{A}_{k}, \hat{b}_{k}\right)\right)
$$

Where \# denotes left inverse. $b_{1}^{\#}$ should be chosen such that $b_{1}^{\#} b_{0} \neq 0$. Then:

$$
f\left(\hat{A}_{k}, \hat{b}_{k}\right)=\frac{1}{b_{i}^{*} b_{0}}\left(g_{k}^{i}+b_{i}^{\#}\left(A_{i}-A_{0}\right)\right)
$$

Then for every $i \in\{1, \ldots, p\}$ we have by Theorem 4.10 that:

$$
\begin{aligned}
\lim _{k \rightarrow \infty}\left\|\left(g_{k}-g^{\prime}\right) x_{k}^{*}\right\| & =\lim _{k \rightarrow \infty} \|\left[b_{i}^{*}\left(A_{0}-A_{i}+b_{0} f\left(\hat{A}_{k}, \hat{b}_{k}\right)-\left(A_{0}-A_{i}+b_{0} f\left(A_{0}, b_{0}\right)\right)\right] x_{k}^{*} \|\right. \\
& =\lim _{k \rightarrow \infty}\left\|b_{i}^{\#} b_{0}\left(f\left(\hat{A}_{k}, \hat{b}_{k}\right)-f\left(A_{0}, b_{0}\right)\right) x_{k}^{*}\right\|=0 .
\end{aligned}
$$

Since by construction $\left|g_{1} x_{s_{k}}^{*}\right|>\epsilon$, for $k$ sufficiently large, we conclude that:

$$
\lim _{k \rightarrow \infty}\left|\frac{g_{s_{i}^{i}}^{i}}{g^{i} x_{s_{k}^{\prime}}}-1\right|=\lim _{k \rightarrow \infty}\left|\frac{\left(g_{s_{k}^{i}}^{i}-g^{i}\right) x_{s_{i}^{*}}^{*}}{g^{i} x_{s_{k}^{*}}^{*}}\right|=0 .
$$

Define:

$$
\alpha_{s_{i}^{\prime}}^{i}:=\frac{g_{s_{i}^{\prime}}^{i} x_{s_{i}^{\prime}}}{g^{\prime} x_{s_{i}^{\prime}}} \text { then: } \lim _{k \rightarrow \infty} \alpha_{s_{k}^{\prime}}^{i}=1
$$

Hence:

$$
\begin{aligned}
f\left(\hat{A}_{s_{i}^{\prime}}, \hat{b}_{s_{i}^{\prime}}\right) x_{s_{i}^{\prime}} & =\frac{1}{b_{i}^{\#} b_{0}}\left(g_{s_{i}^{\prime}}^{i}+b_{i}^{\#}\left(A_{i}-A_{0}\right)\right) x_{s_{i}^{\prime}} \\
& =\frac{1}{b_{i}^{\#} b_{0}}\left(\alpha_{s_{i}^{\prime}}^{i} g^{i}+b_{i}^{\#}\left(A_{i}-A_{0}\right)\right) x_{s_{i}^{\prime}} \\
& =\frac{1}{b_{i}^{\#} b_{0}}\left(\alpha_{s_{i}^{\prime}}^{i} b_{i}^{\#}\left(A_{0}+b_{0} f\left(A_{0}, b_{0}\right)-A_{i}\right)+b_{i}^{\#}\left(A_{i}-A_{0}\right)\right) x_{s_{s}^{\prime}} \\
& =\frac{1}{b_{i}^{\#} b_{0}}\left(\left(\alpha_{s_{i}^{\prime}}^{i}-1\right)\left(b_{i}^{\#}\left[A_{0}-A_{i}+b_{0} f\left(A_{0}, b_{0}\right)\right]+b_{i}^{\dot{2}} b_{0} f\left(A_{0}, b_{0}\right)\right) x_{s_{i}^{\prime}}\right.
\end{aligned}
$$

Hence define:

$$
\Delta_{s_{4}^{\prime}}:=h_{0} b_{0}^{\#}\left(1-\alpha_{s_{*}^{i}}^{i}\right)\left(A_{0}-A_{i}\right)+\left(1-\alpha_{s_{4}^{i}}^{i}\right) f\left(A_{0}, b_{0}\right)
$$

Because of the properties $a, b$ of the sequences $\left\{s_{k}^{i}\right\}, \Delta_{k}$ is now well defined for every $k$. Since

$$
\begin{aligned}
& \lim _{k \rightarrow \infty} \Delta_{s_{k}^{\prime}}=0, \text { for } i \in\{1, \ldots, p\} \text { we also have: } \\
& \lim _{k \rightarrow \infty} \Delta_{k}=0
\end{aligned}
$$

Moreover:

$$
x_{k+1}=\left(A_{0}+b_{0} f\left(A_{0}, b_{0}\right)+\Delta_{k}\right) x_{k}
$$

This completes the proof.

CoROIIARY 4.11 For all $x \in$ We have: 


$$
\left(A_{0}+b_{0} f\left(A_{0}, b_{0}\right)\right) x \in \text { x }
$$

Proor: This follows immediately from Theorem 4.1 and Lemma 4.3.

Note that the above results are valid whether or not $\Lambda$ is contained in the unit disk. But of course for stability of the closed-loop system it is needed that $\Lambda$ is contained in the unit disk.

The theory as presented does not exclude the possibility that $\left(\hat{A}_{k}, \hat{b}_{k}\right)$ or even $f\left(\hat{A}_{h}, \hat{b}_{h}\right)$ does not converge. We have only derived results about their limit points. Indeed it could happen that $\left(\hat{A}_{k}, \hat{b}_{k}\right)$ keeps drifting along a subset of the sphere to which it converges. However this drifting behaviour requires very rare properties of the sequence of estimates. For if it moves too fast it enters the sphere and if moves too slowly it converges. But the question of convergence versus eternal drifting remains relatively unimportant considering Theorem 4.1 .

Simulations. Extensive simulations have been done for low order systems $(n \leqslant 6)$. As could be expected convergence gets slower as $n$ increases. Problems with the reachability of limit points have not been observed and hence it can be expected that the imposed condition is superfluous.

\section{Conclusions.}

An algorithm has been proposed and analysed for adaptive pole placement. A weak form of selftuning has been derived under the reachability condition on the limit points of the estimates. In a forthcoming paper the presented ideas will be applied to a more realistic class of systems, namely SISO systems with unobserved states. There we will also investigate the state trajectory of the controlled system.

\section{RIFERENCES}

1. A. Bi:CKeR, P.R. Kumar, C.Z. Wei (1985). Adaptive control with the stochastic approximation algorithm: geometry and convergence. IEEE Trans. Aut. Contr. AC-30, 330-338.

2. B. MARTENSSON (1985). The order of any stabilizing regulator is sufticient a priori information for adaptive stabilization. Systems \& Control Letters 6, 87-91

3. J.W. Polderman (1986). Adaptive Pole Assignment by State Feedback. Proceedings of the 25th IEEE Conference on Decision and Control, Athens, Greece.

4. J.W. Potdrirman (1985). On the necessity of identifying the true system in adaptive $L Q$ control, to appear in Systems and Control Letters.

5. S.S. SASTRY (1984). Model-Reference Adaptive Control-Stability,Parameter Convergence, and Robustness. IMA Journal of Mathematical Control \& Information 1, $27-66$.

6. W.M. Wonham (1979). Linear Multivariable Control: a Geometric Approach. Springer, New York. 